\title{
Perfil sociodemográfico e padrão de utilização dos serviços de saúde do Sistema Único de Saúde (SUS), 2003- 2008
}

\author{
Socio-demographic profile and utilization patterns \\ of the public healthcare system (SUS), 2003- 2008
}

Zilda Pereira da Silva ${ }^{1}$

Manoel Carlos Sampaio de Almeida Ribeiro ${ }^{2}$

Rita Barradas Barata ${ }^{2}$

Marcia Furquim de Almeida ${ }^{1}$

${ }^{1}$ Departamento de Epidemiologia, Faculdade de Saúde Pública, Universidade de São Paulo. Av Dr Arnaldo 715, Cerqueira César. 01246-904 São Paulo SP. zildapereira@usp.br

${ }^{2}$ Departamento de Medicina

Social, Faculdade de

Ciências Médicas, Santa

Casa de São Paulo
Abstract PNAD data was employed to analyze the utilization profile of health services, and this was measured by the proportion of individuals seeking and reporting use of health services in the prior two weeks and those who reported hospitalization in the preceding 12 months. Private health plans covered $25.9 \%$ of the Brazilian population. Comparing data from 2003 and 2008 surveys, there was no change in the proportion of individuals seeking health services, as well as the proportion of those attended by these services (96\%). The Unified Health System (SUS) was responsible for $56,7 \%$ of all healthcare, providing the bulk of medical visits, vaccine activities and hospital admissions, but accounted for only 1/3 of dental care. There was a reduction in SUS health services utilization with the increase of education and income level, in the two surveys. There was also a decrease in utilization of services due to prevention and an increase in dental problems, accidents, injuries and rehabilitation. The pattern of SUS services utilization per region was inversely related to the proportion of individuals with private health insurance coverage.

Key words Healthcare utilization, Public healthcare system, Private health plans, Reasons for seeking healthcare, Type of healthcare, Type of service
Resumo $O$ objetivo foi analisar a evolução do perfil de utilização de serviços de saúde, entre 2003 e 2008, no Brasil e nas suas macrorregiões. Foram utilizados dados da PNAD. A utilização de serviços de saúde foi medida pela proporção de pessoas que procuraram e foram atendidas nas 2 semanas anteriores e pelos que relataram internação nos últimos 12 meses, segundo SUS e não SUS. Foram analisadas as características socioeconômicas dos usuários, o tipo de atendimento e de serviço e os motivos da procura. A proporção de indivíduos que procuraram serviços de saúde não se alterou, assim como a parcela dos que conseguiram atendimento (96\%), entre 2003 e 2008. O SUS respondeu por $56,7 \%$ dos atendimentos, realizando a maior parte das internações, vacinação e consultas e somente 1/3 das consultas odontológicas. Em 2008, manteve-se o gradiente de redução de utilização de serviços de saúde SUS conforme o aumento de renda e escolaridade. Houve decréscimo da proporção dos que procuraram serviços de saúde para ações de prevenção e aumento de procura para problemas odontológicos, acidentes e lesões e reabilitação. O padrão de utilização do SUS por região esteve inversamente relacionado à proporção de indivíduos com posse de planos privados de saúde.

Palavras-chave Uso de serviços de saúde, SUS, Planos de saúde, Motivo de procura, Tipo de atendimento, Tipo de serviço 


\section{Introdução}

$\mathrm{O}$ acesso à assistência à saúde pode ser medido pela utilização de serviços, contemplando, entre outras, a assistência tanto médica como odontológica, de rotina ou de emergência. A utilização de serviços de saúde é, desta forma, uma expressão positiva do acesso, pois pode impactar na saúde das populações, prevenindo a ocorrência de doenças, reduzindo a mortalidade por causas específicas e aumentando as taxas de sobrevivência. No entanto, a interpretação dos dados sobre utilização de serviços não é trivial, pois a efetiva utilização decorre de uma complexa combinação de fatores, que inclui desde a necessidade, a percepção, as características sociodemográficas e os valores do indivíduo, até a organização e a forma de financiamento do sistema de saúde ${ }^{1,2}$.

Estudos em países com distintos modelos de sistemas de saúde mostram que sua utilização pode variar consideravelmente segundo algumas características dos indivíduos e dos sistemas, como idade, sexo, renda, raça/cor, escolaridade, tipo de cobertura e serviço utilizado ${ }^{2-4}$. Mesmo em países mais desenvolvidos verificam-se iniquidades no acesso ${ }^{4}$. Outro aspecto destas desigualdades que tem sido apontado é a cobertura de serviços odontológicos restrita a alguns grupos ${ }^{5}$.

A organização do sistema de saúde brasileiro está fundada na coexistência de um sistema público - o Sistema Único de Saúde (SUS), orientado pelos princípios de acesso universal e igualitário, sem qualquer condicionalidade -, e de um segmento complementar baseado principalmente na oferta de seguros e de planos de saúde - de adesão voluntária ou mediante benefício trabalhista - e, em menor escala, pelo pagamento direto no ato do consumo do serviço. O SUS responde pela saúde de cerca de 190 milhões de indivíduos e o segmento de seguros e planos de saúde por 49,2 milhões, representando uma cobertura duplicada para $25,9 \%$ da população ${ }^{6}$.

A geração periódica de informações de base populacional e de âmbito nacional constitui-se numa ferramenta necessária para monitorar o acesso aos serviços, permitindo avaliar as políticas públicas de saúde e fornecer subsídios para o seu aperfeiçoamento. Ademais, fornecem dados para compreender como ocorre a articulação dos sistemas público e privado.

O objetivo desse trabalho foi verificar o perfil de utilização de serviços de saúde no âmbito do SUS, em 2008, e analisar sua evolução em relação a 2003, no Brasil e nas suas macrorregiões.

\section{Método}

Foram utilizados os dados do suplemento Acesso e Utilização de Serviços de Saúde da Pesquisa Nacional por Amostras de Domicílios (PNAD) de 2003 e 2008. A amostra da PNAD é representativa dos domicílios particulares do país, sendo que em 2003 não cobria a área rural da região Norte (exceto Tocantins). A amostra da pesquisa foi obtida em três estágios de seleção: unidades primárias - municípios; unidades secundárias - setores censitários; e unidades terciárias domicílios ${ }^{6}$.

Os questionários de ambas as PNADs indagavam sobre a procura por atendimento relacionado à saúde nas duas últimas semanas, se o mesmo foi efetivado e realizado ou não pelo SUS. Além de indagar sobre internação hospitalar nos últimos 12 meses.

Nesse estudo, a utilização de serviços de saúde foi medida pela proporção de pessoas que procuraram e foram atendidas nas duas semanas anteriores à pesquisa, e pela proporção dos que relataram internação nos últimos 12 meses, classificados segundo atendimento SUS e não-SUS.

Para traçar o perfil dos usuários foram utilizadas as variáveis: sexo, idade, cor da pele (autorreferida), escolaridade, renda familiar per capita, posse de plano de saúde e região de residência. Para estudar a utilização dos serviços foram analisadas as variáveis: tipo de atendimento, tipo de serviço e motivos referidos para a procura.

Os indicadores, e os respectivos intervalos de confiança de $95 \%$, foram obtidos por meio do programa estatístico SPSS versão 17.0, utilizando-se a rotina para planos complexos de amostragem, a qual permite a incorporação de estratos, conglomerados e pesos amostrais.

\section{Resultados}

Em 2003, a PNAD entrevistou 384.794 indivíduos, sendo que $14,6 \%$ (IC95\%:14,4-14,8\%) procuraram por atendimento por saúde nas duas últimas semanas, e destes 96,4\% (IC95\%: 96,2-96,6\%) foram atendidos. Em 2008 o comportamento foi semelhante, quando 14,5\% (IC95\%: 14,3-14,7\%) dos 391.868 indivíduos entrevistados referiram terem procurado serviços e 96,4\% (IC95\%: 96,2$96,6 \%)$ referiram terem sido atendidos.

Em relação ao perfil sociodemográfico dos indivíduos que procuraram por atendimento em serviços de saúde nas duas últimas semanas, poucas diferenças estatisticamente significantes foram observadas entre 2003 e 2008, e estas são 
pouco relevantes do ponto de vista epidemiológico. As estimativas obtidas mostram, com uma margem de erro menor que $0,8 \%$ para mais e para menos, que de 2003 para 2008 se manteve o perfil de maior procura de serviços por mulheres ( $17 \%$ contra $12 \%$ dos homens), há um aumento de procura em função da idade $(12 \%$ em 0 a 14 anos, $14 \%$ em 15 a 59 anos e $23 \%$ nos acima dos 60 anos) e das pessoas de cor branca ( $16 \%$ contra $14 \%$ dos pretos e pardos).

Houve maior procura por serviços de saúde nos extremos de escolaridade ( 0 a 3 anos e 11 ou mais anos de estudo, $16 \%$ e $15 \%$, respectivamente, contra $14 \%$ nas faixas de escolaridade intermediárias). Por outro lado, a procura por serviços cresceu com o aumento da renda familiar per capita, variando de $12 \%$ a $18 \%$ entre o $1^{\circ}$ e o $5^{\circ}$ quintis de renda, em 2003 e em 2008.

A procura por serviços de saúde foi mais frequente $(19 \%)$ entre os que possuíam plano de saúde do que entre os que não o possuíam (13\%). Não houve diferença quanto ao tipo de plano (privado e servidor público).

O perfil dos motivos referidos para procura dos serviços foi semelhante nas duas pesquisas. Mais de 50\% dos entrevistados referiram ter procurado serviços de saúde por motivo de doença, tanto em 2003 quanto em 2008. O segundo motivo mais frequente de procura de serviços foram as atividades de prevenção (pré-natal, vacinação e outros), tendo sido observado um declínio na proporção de procura. Em 2003, 31,3\% (IC95\%: 30,6-32,1\%) procuraram os serviços, por este motivo, contra 24,7\% (IC95\%: 24,0-24,3\%) em 2008. Houve um aumento da procura por serviços odontológicos, que foi citado por $8,6 \%$ (IC95\%: 8,2-9,60) em 2003 e por 14,3\% (IC95\%: 13,8-14,8\%) em 2008. Ocorreu também um ligeiro aumento na procura para atendimentos de acidentes e lesões e reabilitação no período, passando de 7\% em 2003 para 9\% em 2008.

Entre os indivíduos entrevistados em 2003 e 2008 foram realizados 54.400 e 55.908 atendimentos de saúde, respectivamente. O SUS foi responsável por 58,5\% (IC95\%: 57,5-59,4\%) destes atendimentos em 2003, e por 56,7\% (IC95\%: 55,957,5\%), em 2008.

Em relação ao perfil sociodemográfico dos que utilizaram o SUS para atendimento em saúde, houve diferença entre 2003 e 2008 apenas em relação à escolaridade e à posse de plano de saúde privado (Tabela 1). Não houve diferença estatisticamente significante na proporção de usuários de serviços de saúde SUS segundo sexo. Também não se observou mudança do padrão de procura de serviços segundo idade, manten- do-se proporção mais elevada de procura entre os mais jovens e idosos. Manteve-se também a utilização mais frequente dos serviços SUS para pretos e pardos, que referiram utilizar estes serviços 1,4 vezes mais que os brancos, embora de 2003 para 2008 tenha ocorrido uma pequena queda na proporção de pretos e pardos que utilizam o SUS (Tabela 1).

O gradiente de redução de utilização de serviços de saúde SUS com o aumento de renda e escolaridade se manteve, mas comparando-se as duas pesquisas há um aumento da proporção de usuários SUS nos grupos de escolaridade mais elevada (de 8 a 10 anos e de 11 anos e mais) estatisticamente significante de 2003 para 2008 (Tabela 1).

Como era de se esperar, a utilização do SUS foi maior dentre aqueles que não possuem planos de saúde. No entanto, a proporção desta utilização sofreu uma pequena queda de 2003 para 2008. Por outro lado, aqueles indivíduos que possuíam planos de saúde privado utilizaram o SUS em uma proporção um pouco maior em 2008. Convém lembrar que 75,4\% (IC95\% 74,976,0\%) dos entrevistados em 2003 não possuíam planos de saúde e que esta proporção sofreu ligeira queda em 2008, passando para $74,1 \%$ (IC95\% 73,5-74,6\%) (Tabela 1).

A análise estratificada avaliando a utilização do SUS segundo escolaridade e posse de plano de saúde privado revelou que houve um aumento estatisticamente significante na utilização do SUS, de 2003 para 2008, nos indivíduos de maior escolaridade, especificamente para aqueles que possuíam planos de saúde privados (resultados não mostrados).

Em 2003, 26.956 indivíduos, isto é, 7,0\%, referiram internação nos últimos 12 meses, destes $67,5 \%$ foram internados no SUS. Resultado semelhante foi observado em 2008, onde 27.878 internações foram referidas, correspondendo a $7,1 \%$ dos entrevistados, e destes $68,3 \%$ foram internados no SUS. Não houve diferença entre 2003 e 2008 (Tabela 2).

Com relação ao tipo de serviço de saúde utilizado há diferenças entre os usuários SUS e não SUS. O SUS foi responsável pela maioria das consultas médicas $(61 \%)$ e dos atendimentos de enfermagem e vacinação (86\%), em 2008. Com relação aos exames subsidiários, o SUS responde por mais da metade do uso destes serviços (57\%). Já as consultas odontológicas ocorrem predominantemente nos serviços não SUS. Não se observaram diferenças estatisticamente significantes no uso desses serviços entre as duas pesquisas (Tabela 2). 
Tabela 1. Perfil dos indivíduos que utilizaram o SUS para atendimento nas duas últimas semanas, Brasil, PNAD 2003 e 2008.

\begin{tabular}{|c|c|c|c|c|c|c|}
\hline \multirow[b]{2}{*}{ Características } & \multicolumn{3}{|c|}{ Utilizaram o SUS em 2003} & \multicolumn{3}{|c|}{ Utilizaram o SUS em 2008} \\
\hline & $\mathrm{N}^{\circ}$ & $\%$ & IC95\% & $\mathrm{N}^{\circ}$ & $\%$ & IC95\% \\
\hline \multicolumn{7}{|l|}{ Sexo } \\
\hline Masculino & 11894 & 58,1 & $57,0-59,2$ & 12241 & 56,3 & $55,2-57,3$ \\
\hline Feminino & 19361 & 58,7 & $57,7-59,6$ & 18860 & 57,0 & $56,1-57,9$ \\
\hline \multicolumn{7}{|l|}{ Idade } \\
\hline $0-14$ & 8671 & 67,5 & $66,2-68,8$ & 7639 & 66,0 & $64,6-67,2$ \\
\hline $15-59$ & 17552 & 54,9 & $54,0-55,9$ & 17113 & 53,7 & $52,8-54,6$ \\
\hline $60 \mathrm{e}+$ & 5022 & 58,0 & $56,5-59,6$ & 5348 & 56,0 & $54,6-57,4$ \\
\hline \multicolumn{7}{|l|}{ Cor } \\
\hline Branca & 14975 & 49,3 & $48,2-50,4$ & 13701 & 47,5 & $46,4-48,6$ \\
\hline Preta e Parda & 16157 & 71,0 & $69,9-72,0$ & 17160 & 67,3 & $66,4-68,2$ \\
\hline \multicolumn{7}{|l|}{ Escolaridade } \\
\hline $0-3$ & 16449 & 73,8 & $72,8-74,7$ & 12539 & 73,0 & $72,1-74,0$ \\
\hline $4-7$ & 8321 & 65,0 & $63,8-66,1$ & 8667 & 66,9 & $65,8-68,0$ \\
\hline $8-10$ & 3248 & 53,5 & $52,0-55,1$ & 4508 & 59,0 & $57,6-60,4$ \\
\hline $11 \mathrm{e}+$ & 314 & 25,8 & $24,7-26,9$ & 5286 & 31,3 & $30,3-32,3$ \\
\hline \multicolumn{7}{|l|}{ Renda Familiar per capita } \\
\hline $1^{\circ}$ quintil & 6993 & 88,8 & $87,6-89,8$ & 6955 & 88,5 & $87,6-89,3$ \\
\hline $2^{\circ}$ quintil & 7292 & 84,1 & $83,1-85,2$ & 7244 & 80,2 & $79,1-81,3$ \\
\hline $3^{\circ}$ quintil & 6882 & 70,9 & $65,5-72,2$ & 6529 & 67,3 & $66,0-68,7$ \\
\hline $4^{\mathrm{o}}$ quintil & 6676 & 56,0 & $54,7-57,3$ & 6886 & 54,3 & $53,2-55,5$ \\
\hline $5^{\circ}$ quintil & 2828 & 20,3 & $19,3-21,3$ & 2781 & 20,2 & $19,2-21,2$ \\
\hline \multicolumn{7}{|l|}{ Plano de Saúde } \\
\hline Não & 28985 & 82,8 & $82,0-83,5$ & 28523 & 79,9 & $79,3-80,6$ \\
\hline Sim, servidor público & 567 & 15,4 & $14,0-17,0$ & 604 & 13,8 & $12,5-15,1$ \\
\hline Sim, privado & 1702 & 11,5 & $10,8-12,3$ & 1974 & 13,4 & $12,6-14,2$ \\
\hline
\end{tabular}

Obs: em negrito são apontadas as diferenças entre 2003 e 2008.

Tabela 2. Proporção de utilização do SUS segundo internação nos últimos 12 meses e tipo de atendimento e de serviços utilizados nas 2 últimas semanas, Brasil, PNAD 2003 e 2008.

\begin{tabular}{|c|c|c|c|c|c|c|}
\hline \multirow{2}{*}{$\begin{array}{l}\text { Tipo de atendimento } \\
\text { e tipo de serviço }\end{array}$} & \multicolumn{3}{|c|}{ Utilizaram SUS em 2003} & \multicolumn{3}{|c|}{ Utilizaram SUS em 2008} \\
\hline & No & $\%$ & IC95\% & No & $\%$ & IC95\% \\
\hline Internação nos últimos 12 meses & 18214 & 67,5 & $66,5-68,5$ & 19045 & 68,3 & $67,4-69,2$ \\
\hline \multicolumn{7}{|l|}{ Atendimento nas últimas 2 semanas } \\
\hline Consulta Médica & 23798 & 61,4 & $60,5-62,4$ & 23812 & 61,3 & $60,4-62,2$ \\
\hline Consulta Odontológica & 1364 & 30,0 & $28,0-32,2$ & 2327 & 29,6 & $28,0-31,1$ \\
\hline Vacinação e atendimento de enfermagem* & 1831 & 82,6 & $80,3-84,6$ & 1509 & 86,2 & $84,1-88,1$ \\
\hline Exames & 1936 & 56,7 & $54,4-58,9$ & 1248 & 57,5 & $55,0-60,0$ \\
\hline Consulta outros profissionais & 421 & 45,4 & $41,5-49,3$ & 503 & 50,3 & $46,7-54,0$ \\
\hline Outros & 2004 & 52,6 & $50,4-54,8$ & 1699 & 53,0 & $50,7-55,3$ \\
\hline \multicolumn{7}{|l|}{ Tipo de Serviço } \\
\hline Ambulatório & 21473 & 54,8 & $53,8-55,9$ & 23492 & 53,8 & $52,9-54,7$ \\
\hline PS/Hospital & 9314 & 74,2 & $72,8-75,6$ & 7234 & 74,1 & $72,7-75,4$ \\
\hline Outros & 464 & 26,4 & $23,5-29,4$ & 375 & 26,9 & $23,8-30,3$ \\
\hline
\end{tabular}

${ }^{*}$ Inclui Vacinação, injeção, curativo, medição de pressão ou outros procedimentos 
Os prontos-socorros e hospitais do SUS foram responsáveis por $2 / 3$ do total de atendimentos. O SUS respondeu também por $54 \%$ dos atendimentos em serviços ambulatoriais, tanto em 2003, quanto em 2008. No entanto, atendimentos em outros serviços foram mais frequentes em usuários não SUS (Tabela 2).

Há diferença dos motivos referidos para a procura por atendimento em serviços de saúde entre usuários SUS e não SUS. De um modo geral, os usuários SUS referiram maior proporção de procura por doenças e acidentes e lesões, correspondendo a praticamente $60 \%$ dos motivos referidos. Por outro lado, para os indivíduos não usuários SUS estes motivos não atingiram 50\% e referiram com maior frequência procura de atendimentos preventivos, para resolver problemas odontológicos e para reabilitação, do que os usuários SUS (Tabela 3).

Comparando-se os resultados das duas pesquisas, observa-se um decréscimo estatisticamente significante da proporção de indivíduos que procuraram os serviços de saúde para atendimentos de prevenção, tanto em usuários SUS como entre os não usuários, e um aumento da procura em função de problemas odontológicos, acidentes e lesões e para reabilitação (Tabela 3).

$\mathrm{O}$ percentual de procura por atendimento e de utilização do SUS, nas duas semanas, foram distintos entre as regiões do país, muito embora, poucas mudanças tenham ocorrido de 2003 para 2008. Cerca de $16 \%$ dos residentes das regiões sudeste e sul procuraram por serviços de saúde, enquanto que foi de $14 \%$ na região centro-oeste, $13 \%$ na nordeste e $11 \%$ na norte.

As regiões norte e nordeste foram as que mais referiram a utilização do SUS, algo próximo a $70 \%$. Num patamar praticamente $20 \%$ mais baixo encontram-se as outras regiões. Pode-se dizer, especificamente em 2008, que a população da região sudeste foi a que em menor proporção utilizou o SUS para atendimento nas duas últimas semanas, seguido de perto pelas regiões sul e centro-oeste. Não houve diferenças entre as regiões entre 2003 e 2008 (Tabela 4). Este padrão de utilização do SUS por região esteve inversamente correlacionado com a posse de planos privados de saúde em 2003 e 2008. Isto é, quanto menor a posse maior a proporção de utilização $(r=0,989$ e $\mathrm{p}=0,001$ para os dados de 2008).

Houve um pequeno aumento da proporção de pessoas com plano de saúde privado na região sudeste de 2003 para 2008, que é estatisticamente significante. Nas demais regiões, isto não se verificou (Tabela 4).

A proporção de utilização do SUS segundo o tipo de atendimento realizado e o tipo de serviço utilizado, nas duas últimas semanas, pouco variou entre 2003 e 2008, não apresentando diferenças estatisticamente significantes. Optou-se, por este fato, pela apresentação na tabela 5 somente dos dados referentes a 2008.

No entanto, entre as regiões, houve diferenças na importância da utilização do SUS conforme o tipo de atendimento. Assim, nas regiões norte e nordeste, o SUS foi responsável pela maior parte das internações (cerca de $80 \%$ ) e das consultas médicas, um pouco mais de $70 \%$. Esta importância foi um pouco menor nas regiões sul (65\% das internações e $59 \%$ das consultas) e centro-oeste (65\% das internações e $62 \%$ das consultas). Por sua vez, no sudeste, o SUS realizou 59\% das internações nos últimos 12 meses e 54\% das consultas médicas nas duas últimas semanas (Tabela 5).

O número absoluto de atendimentos por consultas odontológicas é bem menor do que as médicas, o que repercute no tamanho dos intervalos de confiança, tornando, assim, as estimativas um pouco mais imprecisas. De qualquer modo, o SUS teve uma importância nestas consultas, um pouco maior nas regiões norte e nordeste, quando comparamos com as demais, para

Tabela 3. Motivos referidos para procura de serviços de saúde em usuários e não usuários do SUS, Brasil, PNAD 2003 e 2008.

\begin{tabular}{|c|c|c|c|c|c|c|c|c|c|c|c|c|}
\hline \multirow{2}{*}{$\begin{array}{c}\text { Motivo de } \\
\text { procura }\end{array}$} & \multicolumn{3}{|c|}{ Usuários em 2003} & \multicolumn{3}{|c|}{ Não usuários em 2003} & \multicolumn{3}{|c|}{ Usuários em 2008} & \multicolumn{3}{|c|}{ Não usuários em 2008} \\
\hline & $\mathrm{No}^{\circ}$ & $\%$ & IC95\% & No & $\%$ & IC95\% & No & $\%$ & IC95\% & $\mathrm{N}^{\mathrm{o}}$ & $\%$ & IC95\% \\
\hline Doença & 18101 & 58,4 & $57,5-59,4$ & 9695 & 44,0 & $43,0-45,2$ & 17885 & 58,4 & $57,7-59,4$ & 9480 & 40,9 & $40,0-41,9$ \\
\hline Prevenção* & 9115 & 29,4 & $28,6-30,3$ & 7470 & 34,0 & $33,0-35,0$ & 7166 & 23,5 & $22,7-24,2$ & 6050 & 26,1 & $25,2-27,0$ \\
\hline Prob. Odonto. & 1413 & 4,6 & $4,2-4,9$ & 3114 & 14,2 & $13,4-15,0$ & 2306 & 7,6 & $7,1-8,0$ & 5415 & 23,4 & $22,5-24,3$ \\
\hline Acidentes/lesão & 1720 & 5,6 & $5,3-5,9$ & 1069 & 4,9 & $4,5-5,2$ & 2075 & 6,8 & $6,5-7,1$ & 1251 & 5,4 & $5,1-5,8$ \\
\hline Reabilitação & 611 & 2,0 & $1,8-2,2$ & 647 & 2,9 & $2,7-3,3$ & 1115 & 3,7 & $3,4-4,0$ & 966 & 4,2 & $3,9-4,5$ \\
\hline Total & 30960 & 100,0 & & 21995 & 100,0 & & 30547 & 100,0 & & 23162 & 100,0 & \\
\hline
\end{tabular}

${ }^{*}$ Inclui pré-natal 
Tabela 4. Procura por atendimento nas duas últimas semanas, utilização do SUS e posse de plano de saúde segundo regiões do país, Brasil, PNAD 2003 e 2008.

\begin{tabular}{|c|c|c|c|c|c|c|}
\hline \multirow[b]{2}{*}{ Região } & \multicolumn{2}{|c|}{$\begin{array}{l}\text { Não possui plano } \\
\text { de saúde }\end{array}$} & \multicolumn{2}{|c|}{$\begin{array}{c}\text { Procura por serviço } \\
\text { de saúde }\end{array}$} & \multicolumn{2}{|c|}{$\begin{array}{c}\text { Utilização do } \\
\text { SUS }\end{array}$} \\
\hline & $\%$ & IC95\% & $\%$ & IC95\% & $\%$ & IC95\% \\
\hline \multicolumn{7}{|l|}{ Norte } \\
\hline 2003 & 85,2 & $84,1-86,2$ & 12,4 & $11,7-13,3$ & 68,8 & $66,6-70,9$ \\
\hline 2008 & 86,7 & $85,5-87,8$ & 11,1 & $10,4-11,9$ & 67,7 & $65,2-70,1$ \\
\hline \multicolumn{7}{|l|}{ Nordeste } \\
\hline 2003 & 87,9 & $87,2-88,6$ & 12,9 & $12,4-13,4$ & 71,4 & $69,7-73,0$ \\
\hline 2008 & 86,8 & $86,1-87,5$ & 12,7 & $12,3-13,1$ & 69,1 & $67,5-70,6$ \\
\hline \multicolumn{7}{|l|}{ Sudeste } \\
\hline 2003 & 67,1 & $66,2-68,1$ & 15,7 & $15,3-16,0$ & 52,4 & $51,0-53,8$ \\
\hline 2008 & 64,4 & $63,4-65,3$ & 16,0 & $15,6-16,4$ & 50,0 & $48,7-51,4$ \\
\hline \multicolumn{7}{|l|}{ Sul } \\
\hline 2003 & 72,1 & $70,6-73,5$ & 16,0 & $15,5-16,5$ & 53,9 & $51,7-56,1$ \\
\hline 2008 & 70,0 & $68,5-71,4$ & 16,1 & $15,5-16,7$ & 53,6 & $51,7-55,5$ \\
\hline \multicolumn{7}{|c|}{ Centro-Oeste } \\
\hline 2003 & 75,3 & $73,9-76,5$ & 13,6 & $13,1-14,2$ & 56,6 & $54,1-59,1$ \\
\hline 2008 & 75,4 & $74,1-76,6$ & 13,6 & $13,0-14,2$ & 56,3 & $54,0-58,6$ \\
\hline
\end{tabular}

Obs: em negrito são apontadas as diferenças entre 2003 e 2008.

Tabela 5. Proporção de utilização do SUS segundo internação nos últimos 12 meses e tipo de atendimento e de serviços utilizados nas 2 últimas semanas, por regiões do país, Brasil, PNAD, 2008.

\begin{tabular}{|c|c|c|c|c|c|c|c|c|c|c|}
\hline \multirow{3}{*}{$\begin{array}{l}\text { Tipo de atendimento } \\
\text { e tipo de serviço }\end{array}$} & \multicolumn{10}{|c|}{ Região } \\
\hline & \multicolumn{2}{|c|}{ Norte } & \multicolumn{2}{|c|}{ Nordeste } & \multicolumn{2}{|c|}{ Centro-Oeste } & \multicolumn{2}{|c|}{ Sudeste } & \multicolumn{2}{|r|}{ Sul } \\
\hline & $\%$ & IC95\% & $\%$ & IC95\% & $\%$ & IC95\% & $\%$ & IC95\% & $\%$ & IC95\% \\
\hline $\begin{array}{l}\text { Internação nos últimos } \\
12 \text { meses }\end{array}$ & 78,5 & $75,6-81,1$ & 82,1 & $80,7-83,3$ & 65,0 & $62,9-67,0$ & 58,6 & $57,0-60,2$ & 65,0 & $62,5-67,4$ \\
\hline \multicolumn{11}{|l|}{$\begin{array}{l}\text { Atendimento nas } \\
\text { últimas } 2 \text { semanas }\end{array}$} \\
\hline Consultas Médicas & 72,8 & $70,3-75,2$ & 73,8 & $72,4-75,2$ & 62,4 & $60,1-64,6$ & 54,3 & $52,9-55,8$ & 59,3 & $57,4-61,3$ \\
\hline Consultas & 34,3 & $28,0-41,2$ & 46,4 & $43,1-49,7$ & 24,5 & $20,9-28,9$ & 21,7 & $19,4-24,1$ & 25,3 & $22,2-28,8$ \\
\hline \multicolumn{11}{|l|}{ Odontológicas } \\
\hline $\begin{array}{l}\text { Vacinação e } \\
\text { atendimento de } \\
\text { enfermagem }\end{array}$ & 88,9 & $84,2-92,2$ & 91,8 & $88,8-94,1$ & 90,0 & $83,7-94,1$ & 81,2 & $76,9-84,9$ & 82,2 & $75,2-87,5$ \\
\hline Exames & 70,0 & $61,8-77,0$ & 65,4 & $61,3-69,2$ & 62,4 & $54,7-69,6$ & 50,5 & $46,3-54,7$ & 57,1 & $51,1-62,9$ \\
\hline Outros Profissionais & 68,3 & $59,6-75,9$ & 55,8 & $48,7-62,6$ & 45,3 & $35,1-55,9$ & 47,7 & $42,2-53,3$ & 45,1 & $35,2-55,5$ \\
\hline \multicolumn{11}{|l|}{ Tipo de Serviço } \\
\hline Ambulatório & 66,4 & $63,3-69,3$ & 65,8 & $64,0-67,5$ & 53,9 & $51,3-56,6$ & 47,1 & $45,7-48,5$ & 52,0 & $49,8-54,1$ \\
\hline PS/Hospital & 80,4 & $75,1-84,8$ & 85,0 & $83,2-86,6$ & 72,3 & $68,4-76,0$ & 67,0 & $64,6-69,3$ & 71,4 & $67,4-75,1$ \\
\hline Outros & 21,6 & $14,2-31,5$ & 38,8 & $31,5-46,5$ & 16,4 & $13,3-27,4$ & 23,4 & $18,8-28,7$ & 22,6 & $16,4-30,2$ \\
\hline
\end{tabular}

*Inclui Vacinação, injeção, curativo, medição de pressão ou outros procedimentos.

as quais o setor privado foi ainda mais relevante (Tabela 5).

O SUS foi responsável pela imensa maioria dos atendimentos de vacinação e de outros procedimentos básicos de enfermagem, em todas as regiões do país. Embora haja alguma sobreposição dos intervalos de confiança, os dados sugerem que a importância do SUS seja um pouco maior nas regiões norte, nordeste e centro-oeste do que nas regiões sudeste e sul (Tabela 5). 
Os dados relativos aos exames complementares, por região, demonstram a importância relativa do SUS e sugerem uma associação com percentual da população que possui plano de saúde privado. Assim entre as regiões do país, quanto maior a cobertura da população por plano privado de saúde, menor a importância do SUS na realização $(\mathrm{r}=0,95$ e p=0,012) (Tabela 5).

Ainda em relação ao tipo de atendimento, pode-se constatar que, na região norte, o SUS foi mais relevante que nas demais regiões para a realização de consultas com outros profissionais da saúde (Tabela 5)

Em relação ao tipo de serviço utilizado para o atendimento, em todas as regiões, o SUS teve uma importância relativa maior para os atendimentos em postos de saúde e em hospitais que para os atendimentos realizados em ambulatórios.

\section{Discussão}

O SUS é um sistema que se apoia no conceito de cidadania ao estabelecer como diretriz o acesso universal e integral à atenção à saúde. Se essa característica é uma virtude do sistema brasileiro, tal proposição, no entanto, se constitui no desafio de atender uma população de $190 \mathrm{mi}-$ lhões de pessoas. Estudos mostram que o SUS é o principal financiador da atenção à saúde, especialmente nos níveis extremos de complexidade, como, por exemplo, vacinação na atenção básica e internações na alta complexidade ${ }^{7}$, mas existem áreas onde há insuficiência de atendimento, gerando filas para alguns procedimentos. No momento em que o SUS alcança pouco mais de 20 anos de existência, cada vez mais se torna necessário a geração de conhecimentos, métodos e tecnologia que dêem suporte ao seu pleno desenvolvimento, assim, a investigação sobre a utilização de serviços de saúde do SUS fornece subsídios que podem orientar o desenho das políticas de saúde e a melhoria do desempenho ${ }^{8}$.

Os dados de inquéritos populacionais permitem obter um panorama das necessidades e da utilização dos serviços de saúde nos diversos segmentos socioeconômicos da população. Nesse sentido, a PNAD permite conhecer a cobertura dos serviços de acordo com o tipo de financiamento que as pessoas têm - se elas dependem exclusivamente do sistema público ou se são beneficiárias de planos ou seguros privados de saúde, e como essa condição interfere no uso dos serviços.

A busca de serviços de saúde não é, entretanto, função exclusiva da necessidade, e sim, consequência de diversas determinações que explicam as variações entre grupos sociais, ou entre áreas, e da oferta de serviços. Alguns autores sugerem as seguintes dimensões de análise: condição e necessidade de saúde (morbidade), disponibilidade de médicos, características demográficas, características organizacionais dos serviços de saúde e o modelo de financiamento9.

Este estudo trabalhou apenas com os indivíduos que procuraram atendimento, sem considerar as necessidades de saúde que deram origem à busca desses serviços. Segundo os dados da PNAD, a proporção de indivíduos que procuraram serviços de saúde nas últimas duas semanas não se alterou entre 2003 e 2008, assim como a parcela dos que conseguiram atendimento, mantendo-se num patamar superior a $96 \%$. A alta proporção de pessoas atendidas sugere que as barreiras de acesso não resultam na falta de atendimento, mas não foram trabalhadas informações sobre a agilidade e a oportunidade da atenção prestada, que podem não responder às necessidades de saúde da população.

Em relação ao perfil sociodemográfico, a procura foi maior entre as mulheres e as pessoas de cor branca, assim como há um aumento com o avanço da idade. Os gradientes de utilização de serviços de saúde, segundo escolaridade e renda, observados em 2003, se mantiveram em 2008, mostrando que as condições socioeconômicas estão associadas ao acesso e à utilização dos serviços de saúde. Esse perfil de procura de serviços é semelhante ao observado em outros estudos ${ }^{2,10-12}$.

A procura por serviços de saúde foi mais frequente entre os indivíduos que tinham planos de saúde, quer este fosse privado ou público, o que mostra que sua posse pode levar a um maior acesso a esses serviços. A posse desses planos pode reduzir a existência de possíveis barreiras financeiras no momento do consumo de serviços ${ }^{11}$ ou na potencial redução do tempo de espera para o atendimento. Estudo realizado na região metropolitana de São Paulo mostrou que os não possuidores de planos suportavam maior espera até o atendimento - cerca de 81 minutos, enquanto que os possuidores esperavam 32 minutos, em média ${ }^{13}$. Revisão sistemática sobre fatores relacionados à utilização de serviços de saúde identificou que a posse de plano/seguro de saúde não só incrementa a realização de consultas médicas, como também determina maior utilização de serviços preventivos ${ }^{2}$.

Com relação à utilização de serviços de saúde SUS, mais de $80 \%$ dos usuários destes serviços não possuíam planos de saúde privados ou públicos. Estes dados confirmam que o SUS é o maior agente de financiamento da atenção à saú- 
de do país, e se destaca tanto no provimento de ações básicas como de maior complexidade ${ }^{7}$.

Comparando-se os resultados das pesquisas de 2003 e 2008, observa-se que há uma proporção maior de usuários dos serviços SUS que tinham plano privado de saúde, bem como se observou um incremento de utilização desses serviços entre os indivíduos de elevada escolaridade. Esses dois fenômenos podem estar relacionados entre si, uma vez que beneficiários de planos e seguros privados apresentam maior escolarida$\mathrm{de}^{14}$. A utilização do sistema público por beneficiários de planos de saúde corrobora a ideia de que parcela desses indivíduos não consegue ter acesso integral aos serviços contratados junto às operadoras de planos.

Vale lembrar que os planos de saúde apresentam grande variedade na cobertura de serviços. Segundo estudo realizado pela ANS, com dados da PNAD $2008^{14}$, cerca de 10,4\% dos beneficiários de planos de saúde privados não tinham acesso a internação hospitalar. Mesmo que a normatização instituída em 1998 tenha reduzido as dificuldades de acesso para pessoas com doenças consideradas pré-existentes, ainda existem barreiras tais como deficiência da rede de serviços (insuficiência do apoio diagnóstico terapêutico), inexistência de ofertas específicas (alta complexidade, exames não cobertos, carências) e outros que podem inibir o acesso a tratamentos de alto custo ou de algumas doenças crônicas, que dependendo do plano pode levar seus portadores a recorrer aos serviços do SUS para a realização de tratamento, como indica o estudo de Bahia et al. ${ }^{15}$.

Houve também um crescimento, ainda que discreto, do uso de serviços SUS motivado por acidentes e lesões, de 2003 a 2008. Este resultado é consistente com os dados do SIHSUS (Sistema de Informações Hospitalares) que mostraram um crescimento de 13,5\% nas internações por causas externas, sendo que este foi mais acentuado entre os idosos ${ }^{16}$.

Assim como em 2003, o SUS continuou sendo responsável pela maior parte dos atendimentos em saúde, tanto para consultas médicas, internações e ações preventivas, como nos atendimentos de urgência/emergência. Somente os atendimentos odontológicos foram mais frequentemente relatados por usuários de serviços não SUS. Resultado semelhante foi encontrado nos Estados Unidos, onde pessoas com seguro privado odontológico têm mais visitas ao dentista do que aquelas sem seguro privado ${ }^{17}$.

Ainda que se tenha observado um crescimento de $70,6 \%$ do relato de consultas odontológicas realizadas em serviços do SUS, este cresci- mento foi insuficiente para alterar o padrão de acesso a estes serviços, que continua sendo predominantemente privado. O SUS responde por menos de $1 / 3$ destes atendimentos, situação que não se alterou em relação a 2003.

O aumento da busca de serviços motivada por problemas odontológicos entre usuários SUS e não-SUS pode estar relacionado mais com uma melhora no acesso do que com uma maior necessidade da população. Nos últimos anos temse observado crescente incorporação da saúde bucal no sistema de saúde brasileiro. No período de 2003 a 2008, a ANS identificou um crescimento de $142 \%$ de beneficiários de planos de saúde voltados exclusivamente para o atendimento odontológico $^{14}$. Com relação aos usuários SUS, principalmente no setor público, duas iniciativas colaboraram para ampliação do acesso: a incorporação de ações de saúde bucal nas atividades da Estratégia de Saúde da Família - ESF, no início dos anos 2000, e a Política de Saúde Bucal (Brasil Sorridente) implantada a partir de 2004, que tem como diretriz a reorganização da atenção básica e a ampliação da atenção especializada em saúde bucal ${ }^{18}$. Por fim, apesar desse cenário, é importante considerar que os atendimentos odontológicos apresentam uma maior proporção de pagamento direto, quando comparados com os atendimentos médicos. Viacava ${ }^{19}$ mostrou que, no país, metade das consultas odontológicas foram realizadas mediante desembolso de algum valor, em 2008.

Doença e prevenção continuaram sendo os principais motivos de busca por serviços, em 2003 e 2008. No entanto, doença foi referida com maior frequencia entre os usuários SUS, assim como acidentes e lesão, enquanto que prevenção e problemas odontológicos foram proporcionalmente mais relatados por usuários não-SUS. Esses dados estão de acordo com outros estudos que mostram que grupos populacionais com características socioeconômicas mais desfavoráveis tendem a usar mais os serviços por motivo de doençą ${ }^{20}$.

Por outro lado, se manteve, nas duas pesquisas, a tendência de maior utilização de serviços de saúde preventivos entre os indivíduos com maior escolaridade, renda e com plano de saúde, em consonância com a literatura ${ }^{19-22}$. Estudo que investigou estilos de vida e uso de serviços preventivos para doenças e agravos não-transmissíveis, entre adultos filiados ou não a plano privado de saúde, em Belo Horizonte, concluiu que a realização de exames de dosagem de colesterol, mamografia, papanicolau e determinação da pressão arterial foi significativamente maior entre beneficiários de planos de saúde em compa- 
ração com os que dependiam exclusivamente do SUS, independente de idade e sexo ${ }^{21}$.

As principais alterações ocorridas no período analisado referem-se à diminuição proporcional da busca por atendimentos preventivos e aumento da busca de serviços devido a problemas odontológicos e de reabilitação, tanto no segmento SUS como no não-SUS. Com relação aos atendimentos de prevenção, estes se devem principalmente à procura de serviços de vacinação, incluindo também as atividades de pré-natal e de puericultura. A queda da procura destes serviços provavelmente está relacionada ao declínio sistemático da fecundidade que vem ocorrendo em todas as regiões do país, nas últimas décadas. Em 1980, a taxa de fecundidade total do Brasil era de 4,4 filhos por mulher, valor que decresceu para 1,9, em 2009 23 . Esse patamar, abaixo daquele considerado suficiente para assegurar a reposição populacional, expressa as características do momento atual da transição demográfica brasileira, e se reflete na redução do número absoluto de nascimentos e, consequentemente, na menor demanda por serviços de atenção materno-infantil.

Permaneceram sem grandes alterações as desigualdades regionais existentes no país em relação à procura por atendimento de saúde e de utilização do SUS, no período 2003-2008. A busca por serviço de saúde foi menor na região Norte e maior na Sul e na Sudeste, sendo que a Norte foi a única que registrou pequena retração, provavelmente em razão da incorporação da sua área rural na pesquisa de $2008^{6}$, uma vez que populações que vivem em áreas rurais geralmente têm mais barreiras geográficas de acesso a esses serviços ${ }^{9}$.

Há um padrão típico de utilização de serviços SUS por região no país, as Norte e Nordeste apresentam maior frequência de utilização SUS e a Sudeste a menor proporção, quer segundo tipo de atendimento, quer para o tipo de serviço utilizado. Esse fato está inversamente relacionado à posse de planos de saúde, mostrando que a articulação dos sistemas público e privado ocorre de forma distinta nas cinco macrorregiões. Dados da ANS ${ }^{14}$ mostram que Sul e Sudeste exibem as maiores taxas de cobertura por planos e seguros privados de saúde do país. Além disso, no nosso estudo, a única região que registrou pequeno aumento na posse de planos de saúde foi a Sudeste, o que pode estar relacionado com o aumento do nível de emprego formal, verificado nesse período ${ }^{23}$.

A diferença regional menos pronunciada foi observada nas atividades de vacinação e de atendimento de enfermagem, com uma elevada proporção (entre $80 \%$ e $90 \%$ ) de utilização de serviços SUS em todas as regiões. Parte importante desse conjunto refere-se aos programas de vacinação, que são quase que exclusivamente desenvolvidos em serviços públicos, assim, mesmo a população detentora de planos privados utiliza rotineiramente os serviços SUS para este tipo de procedimento.

Entre os atendimentos analisados, as internações hospitalares apresentaram a segunda maior proporção de atendimento no SUS, em todas as regiões, mostrando também as maiores diferenças inter-regionais. A forte presença do SUS possivelmente seja resultante dos elevados custos envolvidos nesses atendimentos, além do fato de que alguns planos de saúde não cobrem este tipo de atendimento ${ }^{14,15}$. A elevada proporção de atendimento SUS nas regiões norte e nordeste mostra a menor presença da saúde suplementar nessas regiões, bem como expressa diferenças de cobertura desse serviço pelos planos.

Chama atenção a elevada proporção de uso de consultas odontológicas SUS verificada na região Nordeste, quando comparada às demais regiões. Tal fato pode ser explicado, em parte, pela elevada presença do PSF (Programa de Saúde da Família) nesta região (70\% de cobertura) ${ }^{19}$, que na última década passou a incorporar ações de saúde bucal. Por outro lado, a região Sudeste exibe a menor proporção de atendimento odontológico SUS, o que pode estar relacionado com a maior proporção de beneficiários de planos de saúde, incluindo aqueles exclusivamente odontológicos, que cobrem cerca de $11 \%$ da população, o que corresponde ao dobro do observado nas demais regiões ${ }^{14}$. Deve-se considerar também que a população dessa região apresenta maior poder aquisitivo, uma vez que este tipo de atendimento ainda depende, em boa medida, de desembolso direto para sua utilização ${ }^{19}$.

Estudos sobre a procura e a utilização de serviços de saúde podem contribuir para a organização da assistência, uma vez que permitem estabelecer quais os níveis de cobertura, e como estes se diferenciam segundo os atributos dos indivíduos e a distribuição regional, identificando assim grupos populacionais mais vulneráveis ${ }^{12}$. Este estudo analisou um intervalo de tempo relativamente curto e, mesmo assim, captou algumas alterações no perfil dos usuários SUS e no padrão de utilização de serviços, que merecem ser mais bem investigadas para ampliar sua compreensão. Estudos rotineiros são essenciais para o monitoramento do desempenho do sistema de saúde, pois permitem verificar se as novas ações, ou estratégias, implementadas estão alcançando as metas estipuladas e se é necessário produzir intervenções para seu aprimoramento. 


\section{Colaboradores}

Todos os autores participaram da concepção, delineamento, análise de dados e da redação final do artigo.

\section{Referências}

1. Travassos C, Martins M. Uma revisão sobre os conceitos de acesso e utilização de serviços de saúde. Cad Saude Publica 2004; 20(Supl. 2):S190-S198.

2. Mendoza-Sassi R, Béria JU, Barros AJD. Outpatient health service utilization and associated factors: a population-based study. Cad Saude Publica 2003; 37(3):372-378.

3. U.S. Department of Health and Human Services. National Healthcare Disparities Report, 2009. AHRQ Publication 10, march 2010.

4. van Doorslaer E, Masseria C, Koolman X; for the OECD Health Equity Research Group. Inequalities in access to medical care by income in developed countries. CMAJ 2006; 174(2):177-183.

5. Schwarz E. Access to oral health care - an Australian perspective. Community Dentistry and Oral Epidemilogy 2006; 34(3):225-231.

6. Instituto Brasileiro de Geografia e Estatística (IBGE). Pesquisa Nacional por Amostra de Domicílios - um panorama da saúde no Brasil: acesso e utilização dos serviços, condições de saúde e fatores de risco e proteção à saúde 2008. Rio de Janeiro: IBGE; 2010.

7. Porto SM, Santos IS, Uga MAD. A utilização de serviços de saúde por sistema de financiamento. Cien Saude Colet 2006; 11(4):895-910.

8. Travassos C, Novaes HMD. Investigação e avaliação em serviços de saúde. Cad Saude Publica 2004; 20(Supl. 2):S144-S145.

9. Pinto LF, Soranz DR. Planos privados de assistência à saúde: cobertura populacional no Brasil. Cienc Saude Colet 2004; 9(1):85-98.

10. Pinheiro RJ, Viacava F, Travassos C, Brito AS. Gênero, morbidade, acesso e utilização de serviços de saúde no Brasil. Cienc Saude Colet 2002; 7(4):687-707.

11. Travassos C, Oliveira EXG, Viacava F. Desigualdades geográficas e sociais no aceso aos serviços de saúde no Brasil: 1998e 2003. Cien Saude Colet 2006, 11(4):975-986.

12. Dias-da-Costa JS, Gigante DP, Horta BL, Barros FC, Victora CG. Utilização de serviços de saúde por adultos da coorte de nascimentos de 1982 a 2004-5, Pelotas, RS. Rev Saude Publica 2008; 42(Supl. 2):51-59.

13. Pessoto UC, Heimann LH, Boaretto RC, Castro IEC, Kayano J, Ibanhes LC, Junqueira V, Rocha JL, Barboza R, Cortizo CT, Martins LC, Luiz OC. Desigualdades no acesso e utilização dos serviços de saúde na Região Metropolitana de São Paulo. Cienc Saude Colet 2007 12(2):351-362.

14. Agência Nacional de Saúde Suplementar (ANS). Caderno de Informações da Saúde Suplementar: beneficiários, operadoras e planos. Rio de Janeiro: ANS; 2010.
15. Bahia L, Simmer E, Oliveira DC. Cobertura de planos privados de saúde e doenças crônicas: notas sobre utilização de procedimentos de alto custo. Cien Saude Colet 2004; 9(4):921-929.

16. Rede Interagencial de Informações para a Saúde RIPSA. Indicadores e Dados Básicos para a Saúde IDB 2008. [acessado 2011 jan 14]. Disponível em: http://tabnet.datasus.gov.br/cgi/tabcgi.exe?idb2009/ d14.def

17. Bloom B, Cohen RA. Dental insurance for persons under 65 age years with private health insurance: United States, 2008. NCHS Data Brief 2010; 40:1-8.

18. Ministério da Saúde (MS). Brasil Sorridente. [acessado 2011 jan 15]. Disponível em http://portal.saude. gov.br/portal/saude/visualizar_texto.cfm?idtxt= 21125

19. Viacava F. Acesso e uso de serviços pelos brasileiros. Radis, Comunicação em Saúde 2010; 96:12-19.

20. Ribeiro MCSA, Barata RB, Almeida MF, Silva ZP. Perfil sociodemográfico e padrão de utilização de serviços de saúde para usuários e não-usuários do SUS - PNAD 2003. Cien Saude Colet 2006; 11(4):10111022.

21. Lima-Costa MF. Estilos de vida e uso de serviços preventivos de saúde entre adultos filiados ou não a plano privado de saúde (inquérito de saúde de Belo Horizonte). Cien Saude Colet 2004; 9(4):857-864.

22. Camargo MB, Dumith SC, Barros AJD. Uso regular de serviços odontológicos entre adultos: padrões de utilização e tipos de serviços. Cad Saude Publica 2009; 25(9):1894-1906.

23. Instituto Brasileiro de Geografia e Estatística (IBGE). Sintese de Indicadores Sociais - uma análise das condições de vida da população brasileira 2010. Rio de Janeiro: IBGE; 2010.

Artigo apresentado em 21/01/2011

Aprovado em 05/04/2011

Versão final apresentada em 10/05/2011 